\title{
Treatment of COVID-19 with MSCs: how does it work?
}

\author{
Armand Keating* \\ Department of Hematology, Princess Margaret Cancer Centre, University of Toronto, Toronto ON M5G 2M9, Canada
}

Received October 21, 2021; accepted October 27, 2021; published online November 5, 2021

Citation: Keating, A. Treatment of COVID-19 with MSCs: how does it work? Sci China Life Sci, 2022, 65: 849-850, https://doi.org/10.1007/s11427-021-2022-5

Despite the remarkably rapid development and deployment of effective vaccines against the severe acute respiratory syndrome coronavirus 2 (SARS-CoV-2), many patients, especially the unvaccinated, continue to experience severe disease, require ICU care and die of coronavirus disease 2019 (COVID-19). Finding effective treatment for this disease is therefore of considerable importance globally. Numerous strategies, including monoclonal antibodies and antiviral drugs, have been undertaken. Cellular therapy has also been explored, especially with mesenchymal stromal cells (MSCs), which are known to exhibit anti-inflammatory and potential regenerative properties (Keating, 2012). Indeed, a pilot study by Zhao's group of allogeneic MSCs in patients with moderate to severe COVID disease was the first to show promising results, including safety (Leng et al., 2020). Over 80 clinical trials involving MSCs from different sources are now registered in Clinical Trial.gov (https://clinicaltrials. gov/) and most of the studies are ongoing or not yet reported. The challenge in the field has been to understand more fully the mechanisms of MSC action in COVID disease.

In a recent publication in Cell Research (Zhu et al., 2021), Zhao and colleagues have extended their earlier work to address this important goal and explored extensively, the underlying molecular mechanisms likely to be involved. They studied patients enrolled in a prospective, single-blind, placebo-controlled phase II clinical trial of umbilical cordderived MSCs. The 29 subjects per arm were matched for sex, oxygen use, steroid, antibiotic and antiviral agent therapy. Patients on mechanical ventilation were excluded from the trial. Subjects treated with MSCs had significantly shorter hospital stay and symptom duration, and fewer adverse events compared with the placebo group. The authors evaluated pro-inflammatory markers and found that C-reactive protein and pro-inflammatory cytokines, including IL-1RA, IL-18, IL-5, CXCL-1 were reduced in the MSC group compared with the placebo cohort. They also investigated neutrophil extracellular traps (NETs), web-like structures of chromatin fibers which contribute to immunothrombosis in COVID-19 ARD (Middleton et al., 2020), and found reduced NET-DNA plasma levels in the MSC treated group. A larger study would be needed to determine reduction in thrombotic complications in patients with COVID disease and in that context, evaluating the expression of tissue factor (CD142) on MSCs will be important, given its role in initiating coagulation (Moll et al., 2019).

The composition of circulating immune cells was also modulated by MSCs, and of interest, elevated CD14 monocyte levels associated with the severity of COVID disease were reduced in response to MSC treatment. Single cell RNA sequencing of peripheral blood mononuclear cells identified a subpopulation of circulating cells that increased after MSC administration and expressed VNN2, a cell surface antigen associated with human embryonic stem cells. This surprising finding raises the speculation that extramedullary hematopoiesis may be promoted to subserve hematopoietic differentiation, immune cell augmentation and possibly, tissue repair in response to MSC administration. Further studies are required to explore these interesting observations. Other molecules associated with chemotaxis and cell migration, including L-selectin, were also identified,

*Corresponding author (email: armand.keating@uhn.ca) 
suggesting an additional mechanism for mobilizing immune cells to sites of tissue injury.

The authors investigated key immune cells, including B cells, after MSC treatment and showed that while CD40, CD19 and CD81 were upregulated, inhibitory co-receptors that suppress B cell receptor signaling can also be upregulated, inferring a modulatory role of MSCs, particularly early after administration. These studies have implications for how MSCs affect B cell responses to SARS-CoV-2 infection and possibly, the timing of MSC administration. The authors also evaluated $\mathrm{CD} 28$ expression on $\mathrm{T}$ cells and found significantly increased levels on $\mathrm{CD} 4^{+} \mathrm{T}, \mathrm{CD} 4^{+}$memory $\mathrm{T}$ and regulatory $\mathrm{T}$ cells, among others, early after MSC infusion, suggesting the promotion of $\mathrm{T}$ cell activation. The study was supplemented with a small pilot trial of MSC infusion in older healthy volunteers which showed a persistent increase in the frequency of $\mathrm{CD} 28^{+}$T cells up to 6 months after MSC administration.

Taken together with other experiments reported by the Zhao's group in the paper, MSCs appear safe, show efficacy against SARS-CoV-2 infection, reduce pro-inflammatory mediators, modulate $\mathrm{B}$ and $\mathrm{T}$ cell function, reduce inflammation by several mechanisms, including modulating expression of chemotactic factors, and appear to reduce lung injury.

It is well-established that outcomes of clinical trials with MSCs for a variety of disorders, including the treatment of COVID disease, are variable. The considerable variability observed from trial to trial for the same condition is most likely due to a number of factors, including tissue source, and differences in donor, culture methodology, passage number, cryopreservation techniques and cell dose. For example, there is uncertainty that a similar outcome to this study can be obtained with bone-marrow derived MSCs. A further consideration is the timing of MSC administration in the course of the disease (Shahani and Datta, 2021). The lack of a universally accepted potency assay for MSCs has made comparisons between clinical studies particularly challenging.

Zhao and colleagues have conducted a valuable study showing that umbilical cord-derived MSCs ameliorate moderate to severe COVID disease and potentially reduce intensive care admissions and even deaths. Moreover, several mechanisms have been proffered to explain their mode of action. It is therefore critical for other groups to validate the results of this study and incumbent on all to specify the key variables that affect MSC function, thereby enhancing the feasibility of reproducing these important experiments and data.

Compliance and ethics The author(s) declare that they have no conflict of interest.

\section{References}

Keating, A. (2012). Mesenchymal stromal cells: new directions. Cell Stem Cell 10, 709-716.

Leng, Z., Zhu, R., Hou, W., Feng, Y., Yang, Y., Han, Q., Shan, G., Meng, F., Du, D., Wang, S., et al. (2020). Transplantation of ACE2 ${ }^{-}$mesenchymal stem cells improves the outcome of patients with COVID-19 pneumonia. Aging Dis 11, 216.

Middleton, E.A., He, X.Y., Denorme, F., Campbell, R.A., Ng, D., Salvatore, S.P., Mostyka, M., Baxter-Stoltzfus, A., Borczuk, A.C., Loda, M., et al. (2020). Neutrophil extracellular traps contribute to immunothrombosis in COVID-19 acute respiratory distress syndrome. Blood 136, 1169-1179.

Moll, G., Ankrum, J.A., Kamhieh-Milz, J., Bieback, K., Ringdén, O., Volk, H.D., Geissler, S., and Reinke, P. (2019). Intravascular mesenchymal stromal/stem cell therapy product diversification: time for new clinical guidelines. Trends Mol Med 25, 149-163.

Shahani, P., and Datta, I. (2021). Mesenchymal stromal cell therapy for coronavirus disease 2019: which? when? and how much? Cytotherapy 23, 861-873.

Zhu, R., Yan, T., Feng, Y., Liu, Y., Cao, H., Peng, G., Yang, Y., Xu, Z., Liu, J., Hou, W., et al. (2021). Mesenchymal stem cell treatment improves outcome of COVID-19 patients via multiple immunomodulatory mechanisms. Cell Res doi: 10.1038/s41422-021-00573-y. 